\title{
Applying set membership strategy in state of charge estimation for Lithium-ion battery
}

\author{
WANG JIANHONG ${ }^{1}$, RICARDO A. RAMIREZ-MENDOZA ${ }^{2}$
}

${ }^{1}$ School of Electronic Engineering and Automation, Jiangxi University of Science and Technology, Ganzhou, 343100, CHINA

${ }^{2}$ School of Engineering and Sciences, Tecnologico de Monterrey, Monterrey, Ave. Eugenio Garza Sada 2501, Monterrey, N.L., 64849, MEXICO

\begin{abstract}
As state of charge is one important variable to monitor the later battery management system, and as traditional Kalman filter can be used to estimate the state of charge for Lithium-ion battery on basis of probability distribution on external noise. To relax this strict assumption on external noise, set membership strategy is proposed to achieve our goal in case of unknown but bounded noise. External noise with unknown but bounded is more realistic than white noise. After equivalent circuit model is used to describe the Lithium-ion battery charging and discharging properties, one state space equation is constructed to regard state of charge as its state variable. Based on state space model about state of charge, two kinds of set membership strategies are put forth to achieve the state estimation, which corresponds to state of charge estimation. Due to external noise is bounded, i.e. external noise is in a set, we construct interval and ellipsoid estimation for state estimation respectively in case of external noise is assumed in an interval or ellipsoid. Then midpoint of interval or center of the ellipsoid are chosen as the final value for state of charge estimation. Finally, one simulation example confirms our theoretical results.

Keywords: Lithium-ion battery; State of charge estimation; Set membership; Interval estimation; Ellipsoid estimation
\end{abstract}

Received: May 19, 2021. Revised: December 4, 2021. Accepted: December 25, 2021. Published: January 6, 2022.

\section{Introduction}

Lithium-ion battery is the leading energy storage technology for many fields, such as electric vehicle, modern electric grids, transformation, etc. The main features of Lithium-ion battery include energy density, a long time and a lower self-discharge rate, so many research on these main features of Lithium-ion battery are carried out in recent years from their own different points of view. One interesting area of research is battery state estimation, especially named as state of charge (SOC) estimation, as State of charge can not only reflect the remaining capacity of Lithium0ion battery, but also embody the performance and endurance mileage of electric vehicle. Furthermore State of charge is the most important factor to be used in the battery management system, which is critical for the safety, efficiency and life expectancy of Lithium-ion battery. Generally State of charge indicates the remaining battery capacity to show how long the battery will last. It helps the battery management system to protect the battery from overcharging and over-discharging, and makes energy management system to determine an effective dispatching strategy. But State of charge can not be directly measured using physical sensors, then it must be estimated using some newly developed methods with the aid of measurable signals such as the voltage and current of the battery. Then here in this paper, State of charge estimation is our concerned problem for Lithium-ion battery.

State of charge estimation has been widely studied and lots of estimation algorithms have been proposed to acquire precise state of charge estimation. An improved extended Kalman filter method is presented to estimate state of charge for vanadium redox battery (Mohamed MR, 2015), using a gain factor. Some unknown parameters from state space model are identified by classical least 
squares method. The square root cubature Kalman filter algorithm has been developed to estimate the state of charge of battery (Guarnieri Massimo, 2016), where $2 n$ points are calculated to give the same weight, according to cubature transform to approximate the mean of state variables. To improve the accuracy and reliability of state of charge estimation for battery, an improved adaptive cubature Kalman filter is proposed in (Hong WC, 2015), where the battery model parameters are online identified by the forgetting factor recursive least squares algorithm. An adaptive forgetting recursive least squares method is exploited to optimize the estimation alertness and numerical stability (Petchsingh C, 2016), so as to achieve online adaptation of model parameters. To reduce the iterative computational complexity, a two stage recursive least squares approach is developed to identify the model parameters (Li X, Xiong J, 2018), then the measurement values of the open circuit voltage at varying relaxation periods and three temperatures are sampled to establish the relationships between state of charge and open circuit voltage. In (Ngamsai Kittima, 2015), a multi-scale parameter adaptive method based on dual Kalman filters is applied to estimate multiple parameters. Based on battery circuit model and battery model state equation, the real time recursive least squares method with forgetting factor is used to identify unknown battery parameters (Ressel S, Bill F, 2018). After introducing the concept of state of health, the average error of the obtained state of charge estimation is less than one given value. A novel state and parameter co-estimator is developed to concurrently estimate the state and model parameters of a Thevenin model for Liquid mental battery (Chou YS, Hsu NY, 2016), where the adaptive unscented Kalman filter (UKF) is employed for state estimation, including a battery state of charge. After performing Lithium-ion battery modelling and off-line parameter identification, a sensitivity analysis experiment is designed to verify which model parameter has the greatest influence on state of charge estimation (Zhong Q, Zhong F, 2016).
To improve the state of charge estimation accuracy under uncertain measurement noise statistics, a variational Bayesian approximation based adaptive dual extended Kalman filter is proposed in (Xiong B, Zhao J, 2017), and the measurement noise variances are simultaneously estimated in the state of charge estimation process. Actually to the best of our knowledge, these state of charge estimation methodologies can be roughly divided into data-driven methods and model-based methods (Wei Z, Tseng KJ, 2017). In the model-based methods, the Kalman filter based state of charge estimation methods have the merits of self-correction, online computation, and the availability of dynamic state of charge estimation (Wei Z, Tseng KJ, 2016). Kalman filter was firstly proposed to estimate the state of linear system, then in order to apply it into nonlinear system, the extended Kalman filter (EKF) and unscented Kalman filter were developed. Meanwhile the date-driven methods typically include the look up table method, matching learning based method, artificial neural networks and support vector machine, etc (Wei Z, Bhattaraia A, 2018). the data driven method means that in estimating the state whatever in linear system or nonlinear system, no mathematical model is needed, i.e. the state is constructed only directly by observed data (Lin, C, $\mathrm{Mu}, \mathrm{H}, 2017$ ), so a large number of training data covering all of the operating conditions is collected to improve the estimation accuracy of the considered state.

From above mentioned papers or other literatures, we see that it is only Kalman filter that is used to achieve the state estimation. Here we regard all kinds of Kalman filter's extended forms as the same category. To the best of our knowledge that no other new strategy is proposed to estimate the unknown state, except Kalman filter or its extended forms. Furthermore through understanding Kalman filter for state estimation carefully, roughly speaking, Kalman filter holds for state estimation in case that the considered external noise must be a zero mean random signal, i.e. white and normal noise. This condition corresponds to the classical probabilistic 
description on external noise. But this white noise is an idea case, and it does not exist in reality. To relax this strict probabilistic description on external noise, we propose to apply set membership estimation strategy in estimating our considered state in the presence of unknown but bounded noise. Here our considered unknown but bounded external noise is more realistic than white noise in engineering or other research field. It means that our goal is to estimate the state in case of the unknown but bounded external noise in this paper, where the estimated state corresponds to the state of charge in the constructed state space equation. Due to classical Kalman filter or its extended forms are useless within the framework of unknown but bounded external noise, so it is necessary to propose another estimation strategy to identify unknown state on the basis of unknown but bounded external noise. The idea of set membership estimation is from system identification theory or adaptive control, and in order to apply set membership estimation to deal with the problem of estimate the state of charge for Lithium-ion battery, firstly we need to reformulate one state space equation for the state of charge estimation, through using one equivalent circuit model to replace the considered state of charge estimation for Lithium-ion battery. Based on this constructed state space equation corresponding to the state of charge for Lithium-ion battery, then the idea of set membership estimation strategy can be easily applied here. More specially, due to the external noise is unknown but bounded, i.e. it is assumed to be in one set priori within the whole framework of set membership estimation, then two kinds of set membership estimation strategies are proposed here based on the used set, which includes the external noise. Without loss of generality, according to the commonly used interval and ellipsoid for the external noise, the interval estimation and ellipsoid estimation are derived for the considered state estimation respectively, which corresponds to the state of charge estimation for Lithium-ion battery. This correspondence is from the equivalent state space equation. Based on our obtained interval estimation or ellipsoid estimation, the midpoint of the interval estimation can be chosen as the final state estimation and similarly the center of the ellipsoid estimation can be also chosen.

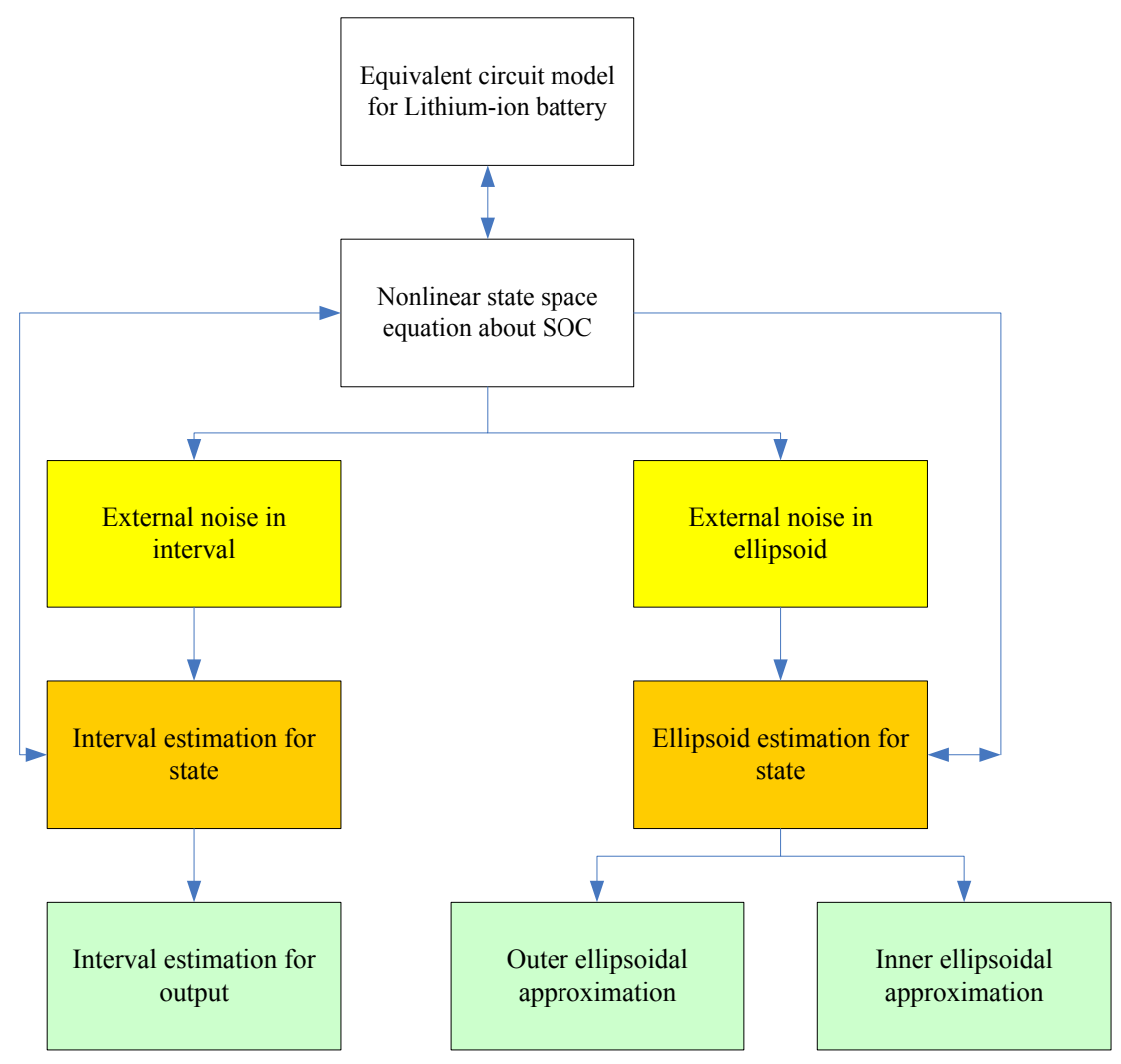


Figure 1 A flowchart of our paper

The paper is organized as follows. In section 2, the battery modelling is addressed, further the definition of state of charge and the state space models for state of charge estimation are also described. In section 3, consider the external noise is in one interval, which is a special case of the unknown but bounded, then the interval estimation for the state is derived at every time instant by our own mathematical derivation. Furthermore, for completeness, the interval estimation for the output is also obtained, though it is not our concern. In section 4, consider the external noise is in one ellipsoid, then we investigate to build ellipsoidal approximation of the state estimation. The main contribution in this section 4 is that given two ellipsoids, we need to find the best inner and outer ellipsoidal approximations of their arithmetic sum. In section 5, one numerical example illustrates the effectiveness of our proposed set membership estimation in estimating the state of charge for Lithium-ion battery. Section 8 ends the paper with final conclusion and points out the next topic.

A flowchart of our two proposed set membership strategies is given in Figure 1, where the yellow parts are our two considered cases of external noise with interval or ellipsoid. The main contributions in these paper is to derive the interval estimation and ellipsoid estimation for state, which correspond to the above two considered unknown but bounded noises respectively.

\section{Battery modelling}

Our considered Lithium-ion battery has some merits in energy density and life, further it is the leading development direction of power batteries for electric vehicles in the future. In order to give a brief introduction on Lithium-ion battery, the internal states of Lithium-ion battery are always divided as four parts, i.e. SOC, temperature, rate of current, state of health. These four states reflect the internal relations of Lithium-ion battery with time variable. Here our emphasis is on the internal structure of Lithium-ion battery, which is shown in Figure 2, whose cell generally comprises four parts: a polymer positive electrode, a diaphragm, a negative electrode and an electrolyte. The positive electrode of Lithium-ion battery is generally composed of Lithium-ion polymer. Common cathode Lithium-ion polymer materials include lithium phthalate, Lithium-ion phosphate, barium acid strontium, Lithium-ion management, nickel diamond and nickel-nickel aluminum ternary lithium. The diaphragm is in the process of the first charge and discharge of the liquid Lithium-ion battery. The electrode material reacts with the electrolyte at the solid-liquid phase interface to form a passivation layer covering the surface of the electrode material to isolate the electrode and the electrolyte, and the Lithium-ion can finish chemical reaction with the diaphragm.

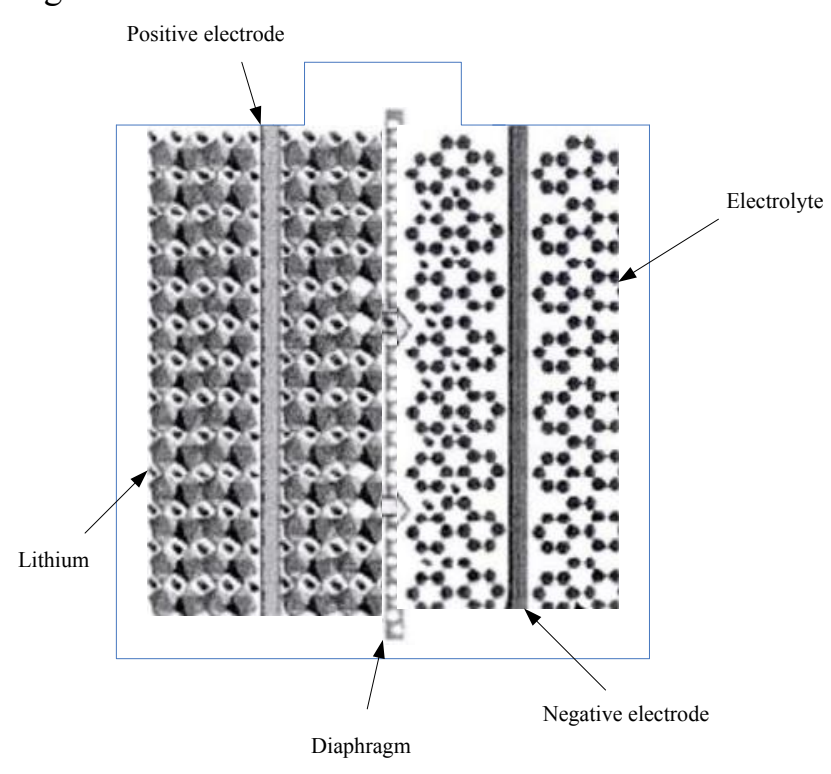

Figure 2. Battery internal structure

Actually in all of literatures on state of charge for Lithium-ion battery, two commonly used battery models exist, i.e. equivalent circuit model and electrochemical model. As electrochemical model is very complex, and it is very difficult to design the latter Kalman filter in case of this electrochemical model, so here in modelling Lithium-ion battery, the equivalent circuit model is recently used. Equivalent circuit model regards the battery internal reactions as a circuit, containing some electronic components, so equivalent circuit model consists of basic circuit components such as resistors, capacitors and voltage 
sources. These four basic circuit components are

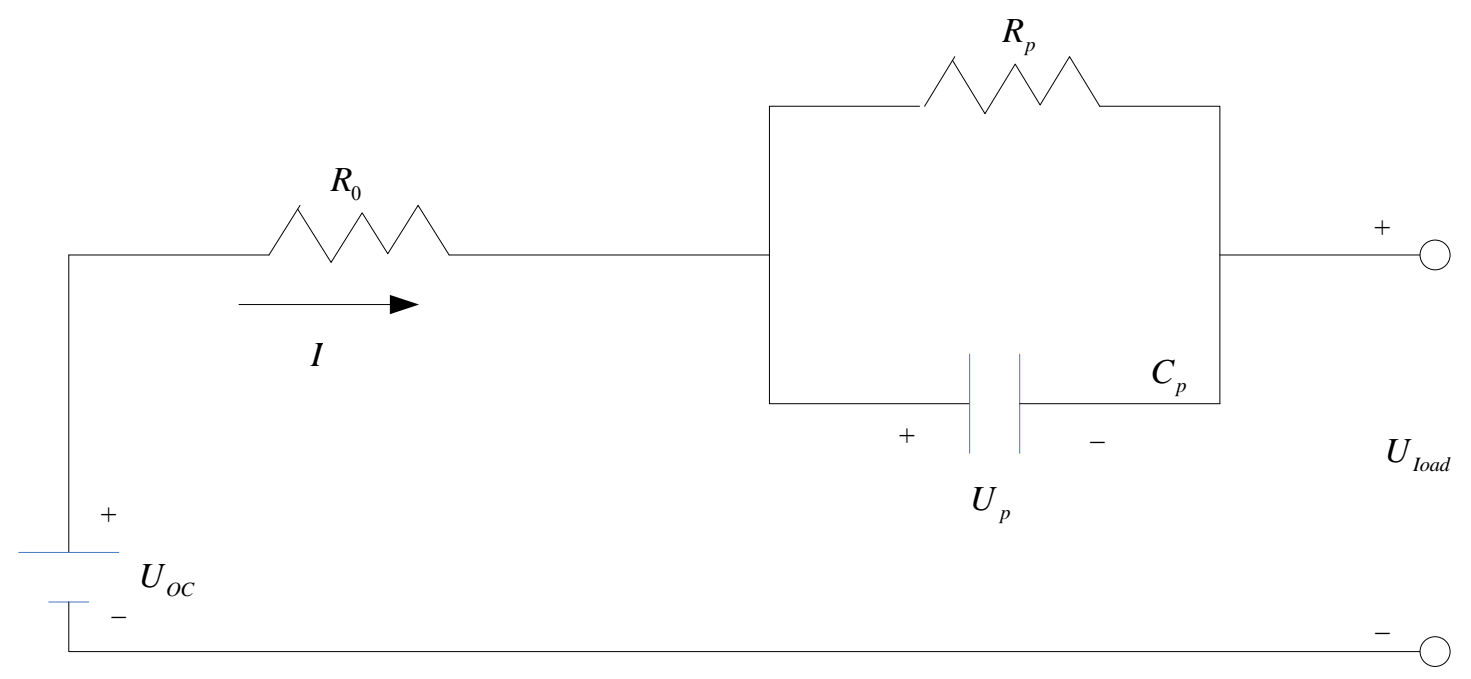

widely explored due to their relatively simple
Figure 3. Equivalent circuit model

where $\left\{d_{i}\right\}_{i=1}^{5}$ are the coefficients of the polynomial form, and $x$ is the state of charge of the battery. The state of charge is defined as a ratio of the remaining capacity over the rated capacity. According to the ampere hour counting method, state of charge can be expressed as follows.

$$
\operatorname{SOC}(t)=\operatorname{SOC}\left(t_{0}\right)-\eta \int_{t_{0}}^{t} \frac{I d t}{Q_{N}}
$$

discharging properties. Through balancing the trade-off between model accuracy and computational complexity, one Thevenin equivalent circuit model is chosen for a Li-ion battery, which is regarded as our battery model

Applying Kirchhoff law, variable $U_{\text {load }}$ is defined as that.

$$
\begin{aligned}
& U_{\text {load }}=U_{O C}-I R_{0}-U_{p} \\
& R_{p} \\
& R_{p}
\end{aligned}
$$

where in equation (1) and (2), $U_{\text {load }}$ is the terminal voltage, $I$ is the load current, $R_{0}$ is the internal ohmic resistance, $R_{p}$ and $C_{p}$ are polarization resistence and polarization capacitance of the battery, $U_{p}$ is the polarization voltage. $U_{O C}$ is the open circuit voltage, which is monotonic with state of charge. Further $U_{O C}$ is rewritten as the following polynomial form.

$$
U_{O C}(x)=d_{5}+d_{4} x+d_{3} x^{2}+d_{2} x^{3}+d_{1} x^{4}
$$

where $k$ is the sample time, $S O C_{k}$ is the statue value at the $k$ th sample time, $T_{s}$ is the specified small sampling period. $U_{O C}\left(S O C_{k}\right)$ denotes a nonlinear function of $\mathrm{SOC}_{k}$.

The parameters in above state space equation (5) and (6) can be identified by classical least squares method, then our goal in this paper is to estimate 
state of charge $\left(S O C_{k}\right)$ at time instant $k$.

\section{Interval estimation for SOC}

In this section we start to apply set membership filter algorithm to estimate the SOC. by combining equation (5) and (6), we see that $S O C_{k}$ at time instant $k$ is one state variable in that state space equation. Furthermore we also want to testify which parameter will influence SOC estimation, then this parameter will be added as the new state variables in the extended state space equation.

\subsection{Preliminary}

As the main model parameter $R_{0}$ is classified as a new state variable with $U_{p}$ and SOC, then an extended state space equation for set membership filter can be given as that.

$$
\begin{gathered}
{\left[\begin{array}{c}
S O C_{k} \\
U_{p, k} \\
R_{0, k}
\end{array}\right]=\left[\begin{array}{ccc}
1 & 0 & 0 \\
0 & \exp \left(-\frac{T_{s}}{R_{p} C_{p}}\right) & 0 \\
0 & 0 & 1
\end{array}\right]\left[\begin{array}{c}
S O C_{k-1} \\
U_{p, k-1} \\
R_{0, k-1}
\end{array}\right]+} \\
{\left[\begin{array}{c}
-\eta \\
R_{p}\left(1-\exp \left(-\frac{T_{s}}{R_{p} C_{p}}\right)\right. \\
0
\end{array}\right] I_{k-1}+\left[\begin{array}{c}
w_{1, k-1} \\
w_{2, k-1} \\
w_{3, k-1}
\end{array}\right]} \\
U_{\text {load }, k}=U_{O C}\left(S_{O} O C_{k}\right)-U_{p, k}-I_{k} R_{0}+v_{k}
\end{gathered}
$$

Observing above equation (7) and (8), the problem of state of charge for Lithium-ion battery is to estimate the first state variable $S O C_{k}$ at every time instant $k$. Due to state of charge for Lithium-ion battery is the first element of the state vector in equation (7), then this problem is similar to the state filtering in the modern control theory. So if the state noise or external noise is a white noise, then the classical Kalman filter can be well applied to deal with the filter problem. But if the probability distribution of the state noise or external noise is unknown, then Kalman filter strategy is useless here, due to the white noise is an idea case in reality. To consider other more general case about the state noise or external noise, the property of the state noise or external noise is unknown but bounded. In this whole paper, our contribution concern on deal with the problem of state estimation in case of unknown but bounded external noise.

Then in order to apply set membership algorithm into above state space equation to estimate the first state variable, we rewrite the above two equations (7) and (8) as follows.

$$
\left\{\begin{array}{l}
x(k+1)=A x(k)+B u(k)+D w(k) \\
y(k)=C x(k)+v(k)
\end{array}\right.
$$

where

$$
\begin{aligned}
& x(k+1)=\left[\begin{array}{c}
S O C_{k} \\
U_{p, k} \\
R_{0, k}
\end{array}\right], A=\left[\begin{array}{ccc}
1 & 0 & 0 \\
0 & \exp \left(-\frac{T_{s}}{R_{p} C_{p}}\right) & 0 \\
0 & 0 & 1
\end{array}\right], \\
& B=\left[\begin{array}{c}
-\eta \\
\left.R_{p}\left(1-\exp \left(-\frac{T_{s}}{R_{p} C_{p}}\right)\right)\right] \\
0
\end{array}\right] \\
& w(k)=\left[\begin{array}{c}
w_{1, k-1} \\
w_{2, k-1} \\
w_{3, k-1}
\end{array}\right], u(k)=I_{k-1}, D=I
\end{aligned}
$$

and

$$
y(k)=U_{\text {load }, k}, C=\frac{d\left(U_{O C}\left(S O C_{k}\right)-U_{p, k}-I_{k} R_{0}\right)}{d\left(S O C_{k}\right)},
$$

where in equation (9) $k$ is time instant, $x(k)$ is the state of this system at time instant $k$ with its initial state $x(0), y(k)$ is the observed output at time instant $k \cdot u(k)$ is the control input, $w(k)$ and $v(k)$ are two unknown but bounded state noise and observed noise respectively. All matrices $A, B, C, D$ are some matrices with compatible dimensions, i.e.

$$
A \in R^{n \times n}, B \in R^{n \times m}, D \in R^{n \times n_{w}}, C \in R^{n_{y} \times n}
$$

Our considered linear discrete time invariant system is one state space equation, whose structure can be seen in Figure 4. 


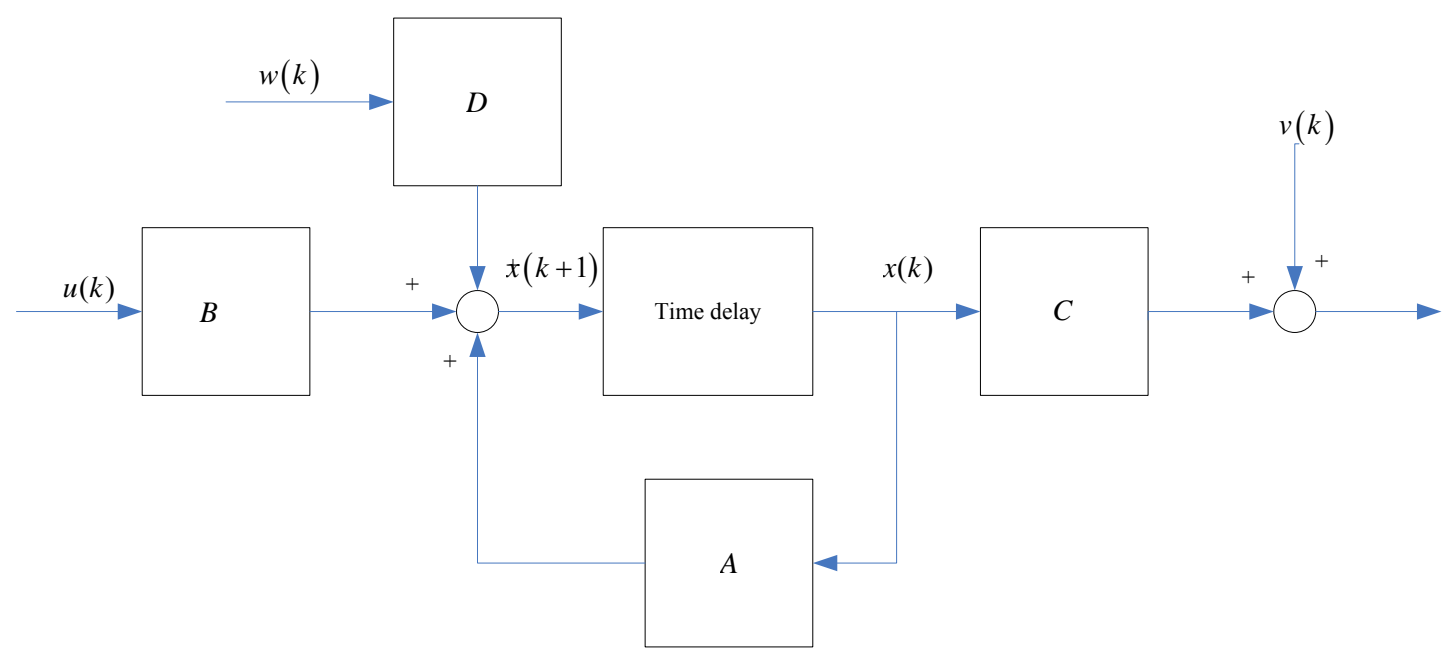

Figure 4 . The structure of state space equation

Let $x$ and $\bar{x}$ be two vectors such that $x \leq \bar{x}$ with the inequality holding componentwise. An interval $[\underline{x}, \bar{x}]$ is defined by.

$$
[\underline{x}, \bar{x}]=\left\{x \in \mathrm{R}^{\mathrm{n}}: \underline{x} \leq x \leq \bar{x}\right\}
$$

Then first of all, we give the assumptions about initial state $x(0)$, state noise $w(k)$ and observed noise $v(k)$.

Assumption 1: There exist three kinds of unknown but bounded signals

$$
[\underline{x}(0), \bar{x}(0)],[\underline{w}(k), \bar{w}(k)],[\underline{v}(k), \bar{v}(k)]
$$

respectively, such that three uncertainties in state space equation (1) be.

$$
\left\{\begin{array}{l}
\underline{x}(0) \leq x(0) \leq \bar{x}(0) \\
\underline{w}(k) \leq w(k) \leq \bar{w}(k) \quad \text { for } \quad \text { all } \quad k \in R^{+} \\
\underline{v}(k) \leq v(k) \leq \bar{v}(k)
\end{array}\right.
$$

where the inequalities are regarded as componentwise.

As interval $[\underline{x}, \bar{x}]$ can not be used in the latter computational process, so its other equivalent form is defined.

Definition 1: The interval $[\underline{x}, \bar{x}]$ can be equivalently represented by the following equivalent form.

$$
C\left(c_{x}, p_{x}\right)=\left\{c_{x}+P_{x} \alpha_{x}: \alpha_{x} \in R^{n},\left\|\alpha_{x}\right\|_{\infty} \leq 1\right\}
$$

where

$$
c_{x}=\frac{\bar{x}+\underline{x}}{2}, P_{x}=\operatorname{diag}\left(p_{x}\right), p_{x}=\frac{\bar{x}-\underline{x}}{2}
$$

Similarly

$$
\bar{x}=c_{x}+p_{x}, \underline{x}=c_{x}+p_{x}
$$

Here the notation \|\|$_{\infty}$ is the infinite norm of one vector, and $\operatorname{diag}()$ is the notation section. Also in Definition $1, c_{x}$ is the center of the interval $[\underline{x}, \bar{x}]$, and $p_{x}$ its radius, i.e. $C\left(c_{x}, p_{x}\right)=[\underline{x}, \bar{x}]$.

Using the above Definition 1, the equivalent forms in Assumption 1 are given as the following Assumption 2.

Assumption 2: There exist three equivalent forms for three intervals

$[\underline{x}(0), \bar{x}(0)],[\underline{w}(k), \bar{w}(k)],[\underline{v}(k), \bar{v}(k)]$ respectively.

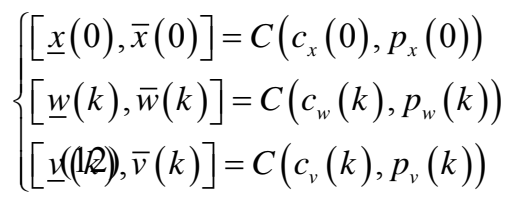

Here the first contribution of our current paper is to construct one interval $[\underline{x}(k), \bar{x}(k)]$ for state estimation $x(k)$ in equation (9), then after substituting $[\underline{x}(k), \bar{x}(k)]$ into the observed equation, the interval $[\underline{y}(k), \bar{y}(k)]$ corresponding to the prediction output can be obtained, while considering three uncertainties about initial state $x(0)$, state noise $w(k)$ and observed noise $v(k)$.

\subsection{Interval estimation}

Firstly to obtain one interval $[\underline{x}(k), \bar{x}(k)]$ for state estimation $x(k)$ at time instant $k$, we take $z$ transformation on both sides of the state equation, i.e.

$$
z X(z)-z x(0)=A X(z)+B U(z)+D W(z)
$$


where $z$ is the variable in frequency domain, and $X(z), U(z), W(z)$ are the transformation results in frequent domain, corresponding to their forms in time domain $x(k), u(k), w(k)$.

Formulating equation (17) to give that.

$$
X(z)=(z I-A)^{-1} z x(0)+(z I-A)^{-1} B U(z)+(z I-A)^{-1} D W(z)
$$

Taking inverse $z$ transformation on both sides of equation (18), it holds that.

$$
\begin{aligned}
x(k) & =A^{k} x(0)+\sum_{i=0}^{k-1} A^{k-i-1} B u(i)+\sum_{i=0}^{k-1} A^{k-i-1} D w(i) \\
& =A^{k} x(0)+\sum_{i=0}^{k-1} A^{k-i-1}(B u(i)+D w(i))
\end{aligned}
$$

Based on equation (19), we proceed to construct intervals for state estimation and prediction output respectively.

Observing equation (19), two uncertainties exist, i.e. initial state $x(0)$ and state noise $w(i)$. From Assumption 2, two intervals about initial state $x(0)$ and state noise $w(i)$ are given as.

$$
\left\{\begin{array}{l}
x(0) \in C\left(c_{x}(0), p_{x}(0)\right) \\
w(i) \in C\left(c_{w}(i), p_{w}(i)\right)
\end{array}\right.
$$

Then we can describe the uncertain initial state $x(0)$ and state noise $w(i)$ by.

$$
\left\{\begin{array}{l}
x(0)=c_{x}(0)+P_{x}(0) \alpha_{x} \\
w(i)=c_{w}(i)+P_{w}(i) \alpha_{w}
\end{array}\right.
$$

where $\alpha_{x} \in R^{n}$ and $\alpha_{w} \in R^{n_{w}}$ such that.

$$
\left\|\alpha_{x}\right\| \leq 1, \text { and }\left\|\alpha_{w}\right\| \leq 1
$$

Substituting equation (20) into $x(k)$, then it holds that.

$$
\begin{aligned}
& x(k)=A^{k}\left[c_{x}(0)+P_{x}(0) \alpha_{x}\right]+\sum_{i=0}^{k-1} A^{k-i-1} B u(i)+ \\
& \begin{aligned}
\sum_{i=0}^{k-1} A^{k-i-1} D\left[c_{w}(i)+P_{w}(i) \alpha_{w}\right] \\
\quad=A^{k} c_{x}(0)+ \\
\sum_{i=0}^{k-1} A^{k-i-1} B u(i)+\sum_{i=0}^{k-1} A^{k-i-1} D c_{w}(i)+A^{k} P_{x}(0) \alpha_{x}+\sum_{i=0}^{k-1} A^{k-i-1} D P_{w}(i) \alpha_{w}
\end{aligned}
\end{aligned}
$$

$$
\left\{\begin{array}{l}
c_{x}(k)=A^{k} c_{x}(0)+\sum_{i=0}^{k-1} A^{k-i-1} B u(i)+\sum_{i=0}^{k-1} A^{k-i-1} D c_{w}(i) \\
p_{x}(k)=\left|A^{k}\right| p_{x}(0)+\sum_{i=0}^{k-1}\left|A^{k-i-1} D\right| p_{w}(i)
\end{array}\right.
$$

where notation || is the absolute value.

Then it holds that.

$$
x(k) \in C\left(c_{x}(k), p_{x}(k)\right), k=1,2 \cdots N
$$

Generally the above derivations can be formulated as the following Theorem 1.

Theorem 1: Set $C\left(c_{x}(0), p_{x}(0)\right) \quad$ and $C\left(c_{w}(i), p_{w}(i)\right)$ be center-radius representations of two uncertainties $x(0)$ and $w(i)$, the interval $[\underline{x}(k), \bar{x}(k)]$ for state estimation $x(k)$ in state space equation (1) is constructed as.

$$
\left\{\begin{aligned}
\underline{x}(k) & =c_{x}(k)-p_{x}(k) \\
& =A^{k} c_{x}(0)+\sum_{i=0}^{k-1} A^{k-i-1} B u(i)+\sum_{i=0}^{k-1} A^{k-i-1} D c_{w}(i)-\left|A^{k}\right| p_{x}(0) \\
-\sum_{i=0}^{k-1} \mid & A^{k-i-1} D \mid p_{w}(i) \\
\bar{x}(k) & =c_{x}(k)+p_{x}(k) \\
& =A^{k} c_{x}(0)+\sum_{i=0}^{k-1} A^{k-i-1} B u(i)+\sum_{i=0}^{k-1} A^{k-i-1} D c_{w}(i)+\left|A^{k}\right| p_{x}(0) \\
+\sum_{i=0}^{k-1} \mid & A^{k-i-1} D \mid p_{w}(i)
\end{aligned}\right.
$$

where $c_{x}(k)$ and $p_{x}(k)$ are defined in equation (24), then interval for state estimation is given that.

$$
x(k) \in C\left(c_{x}(k), p_{x}(k)\right)=[\underline{x}(k), \bar{x}(k)], k=1,2 \cdots N
$$

To analyze the recursive relation between the $k$ th interval $[\underline{x}(k), \bar{x}(k)]$ and its latter $k+1$ th interval $[\underline{x}(k+1), \bar{x}(k+1)]$, we list their centers as follows.

$$
\left\{\begin{array}{l}
c_{x}(k)=A^{k} c_{x}(0)+\sum_{i=0}^{k-1} A^{k-i-1} B u(i)+\sum_{i=0}^{k-1} A^{k-i-1} D c_{w}(i) \\
c_{x}(k+1)=A^{k+1} c_{x}(0)+\sum_{i=0}^{k} A^{k-i-1} B u(i)+\sum_{i=0}^{k} A^{k-i-1} D c_{w}(i)
\end{array}\right.
$$

Taking subtract operation, we find that.

Define the following pair $C\left(c_{x}(k), p_{x}(k)\right)$ as. 
$c_{x}(k+1)-c_{x}(k)=A^{k} c_{x}(0)(A-I)+\sum_{i=0}^{k-1} A^{k-i-1} B u(i)(A-I)+\quad$ radius is that. $\quad p_{x}(k+1)=|A| p_{x}(k)+|B| p_{w}(k)$

$\sum_{i=0}^{k-1} A^{k-i-1} D c_{w}(i)(A-I)+B u(k)+D c_{w}(k)$

From these two recursive relations between the $=\left[A^{k} c_{x}(0)+\sum_{i=0}^{k-1} A^{k-i-1} B u(i)+\sum_{i=0}^{k-1} A^{k-i-1} D c_{w}(i)\right](A-I)_{k+1 \text { th interval can be obtained from the } k \text { th }}^{\text {adjacent interval for state estimation, we see that the }}$ $+B u(k)+D c_{w}(k)$ interval and the knowledge of control input and state $=c_{x}(k)(A-I)+B u(k)+D c_{w}(k)$

Then it holds that noise. The recursive computation for the interval for state estimation is seen in Figure 5.

$$
c_{x}(k+1)=A c_{x}(k)+B u(k)+D c_{w}(k)
$$

Equation (30) is the recursive expression of the centers. Similarly the recursive expression of the

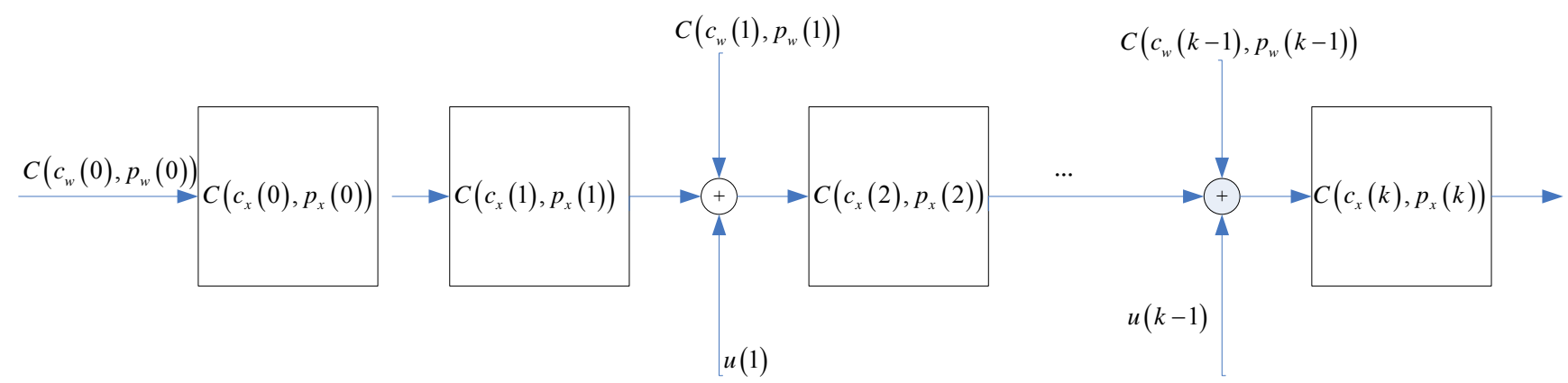

Figure 5. Recursive computation for interval 
But the most important element in model predictive control is the prediction output, so the interval for the prediction output can be obtained by substituting interval $[\underline{x}(k), \bar{x}(k)]$ into the observed equation.

Due to $\quad x(k) \in C\left(c_{x}(k), p_{x}(k)\right) \quad$ and $v(k) \in C\left(c_{v}(k), p_{v}(k)\right)$, i.e.

$$
\left\{\begin{array}{l}
x(k)=c_{x}(k)+P_{x}(k) \alpha_{x} \\
v(k)=c_{v}(k)+P_{v}(k) \alpha_{v}
\end{array}\right.
$$

where $\alpha_{x} \in R^{n}$ and $\alpha_{v} \in R^{n_{v}}$ such that.

$$
\left\|\alpha_{x}\right\| \leq 1 \text {, and }\left\|\alpha_{v}\right\| \leq 1
$$

Substituting equation (32) into the observed equation (9), we have that.

$$
\begin{aligned}
& y(k)=C\left\lfloor c_{x}(k)+P_{x}(k) \alpha_{x}\right\rfloor+\left\lfloor c_{v}(k)+P_{v}(k) \alpha_{v}\right\rfloor= \\
& C c(k)+c(k)+C P_{x}(k) \alpha_{x}+P_{v}(k) \alpha_{v} \\
& c_{y}(k)
\end{aligned}
$$

Similarly define the center and radius as .

$$
\left\{\begin{array}{l}
c_{y}(k)=C c_{x}(k)+c_{v}(k) \\
p_{y}(k)=C p_{x}(k)+p_{v}(k)
\end{array}\right.
$$

Then we have that

Furthermore

$$
y(k) \in C\left(c_{y}(k), p_{y}(k)\right)
$$

$$
\left\{\begin{aligned}
\underline{y}(k) & =c_{y}(k)-p_{y}(k)=C c_{x}(k)+c_{v}(k)-C p_{x}(k)-p_{v}(k) \\
& =C\left(c_{x}(k)-p_{x}(k)\right)+c_{v}(k)-p_{v}(k) \\
\bar{y}(k) & =c_{y}(k)+p_{y}(k)=C c_{x}(k)+c_{v}(k)+C p_{x}(k)+p_{v}(k) \\
& =C\left(c_{x}(k)+p_{x}(k)\right)+c_{v}(k)+p_{v}(k)
\end{aligned}\right.
$$

Then it also means that

$$
y(k) \in C\left(c_{y}(k), p_{y}(k)\right)=[\underline{y}(k), \bar{y}(k)]
$$

The above equation (38) is our interval for the prediction output, which will be used for the next robust model predictive control.

In order to simplify the latter exposition in robust model predictive control, we need the explicit form of interval $y(k) \in C\left(c_{y}(k), p_{y}(k)\right)=[\underline{y}(k), \bar{y}(k)]$. To achieve this goal, some notations are introduced here.

$$
\begin{aligned}
& c_{x}(k)=A^{k} c_{x}(0)+\sum_{i=0}^{k-1} A^{k-i-1} B u(i)+\sum_{i=0}^{k-1} A^{k-i-1} D c_{w}(i)= \\
& c_{1}(k)+\sum_{i=0}^{k-1} c_{2}(k-i-1) u(i) \\
& p_{x}(k)=\left|A^{k}\right| p_{x}(0)+\sum_{i=0}^{k-1}\left|A^{k-i-1} D\right| p_{w}(i) \\
& c_{1}(k)=A^{k} c_{x}(0)+\sum_{i=0}^{k-1} A^{k-i-1} D c_{w}(i), c_{2}(k-i-1)=A^{k-i-1} B
\end{aligned}
$$

Substituting notation (39) into the expressions $\underline{y}(k)$ and $\bar{y}(k)$ respectively, we obtain.

$$
\begin{aligned}
\bar{y}(k) & =c_{y}(k)+p_{y}(k)=C c_{x}(k)+c_{v}(k)+C p_{x}(k)+p_{v}(k) \\
& =C\left(c_{x}(k)+p_{x}(k)\right)+c_{v}(k)+p_{v}(k) \\
& =C\left[c_{1}(k)+\sum_{i=0}^{k-1} c_{2}(k-i-1) u(i)\right]+c_{v}(k)+p_{v}(k)+C p_{x}(k) \\
& =a_{1}(k)+\sum_{i=0}^{k-1} a_{2}(k-i-1) u(i)
\end{aligned}
$$

where

$$
\left\{\begin{array}{l}
a_{1}(k)=C c_{1}(k)+c_{v}(k)+p_{v}(k)+C p_{x}(k) \\
a_{2}(k-i-1)=C c_{2}(k-i-1)
\end{array}\right.
$$

Similarly

$$
\begin{aligned}
& \underline{y}(k)=C\left(c_{x}(k)-p_{x}(k)\right)+c_{v}(k)-p_{v}(k)= \\
& a_{3}(k)+\sum_{i=0}^{k-1} a_{2}(k-i-1) u(i)
\end{aligned}
$$

where

$$
a_{3}(k)=C c_{1}(k)+c_{v}(k)-p_{v}(k)-C p_{x}(k)
$$

The advantage of reformulating $y(k)$ and $\bar{y}(k)$ is that the explicit form can be divided as one linear affine function of the control input $u(i)$. Based on equation (40) and (43), we rewrite equation (38) as.

$$
\begin{aligned}
y(k) & \in C\left(c_{y}(k), p_{y}(k)\right)=[\underline{y}(k), \bar{y}(k)] \\
& =\left[a_{3}(k)+\sum_{i=0}^{k-1} a_{2}(k-i-1) u(i), a_{1}(k)+\sum_{i=0}^{k-1} a_{2}(k-i-1) u(i)\right]
\end{aligned}
$$

Then equation (44) will be used in the detailed computation about the other research field. From equation (32), the center or midpoint $c_{x}(k)$ can be chosen as the final state estimation, corresponding to 
our considered state of charge. Also combing equation (32) and (44), we derive not only the interval estimation for the state, but also the interval estimation for the prediction output. As the emphasis here is only the interval estimation for the state, so that interval estimation for the prediction output is not deep studied here, and it is applied in learning model predictive control.

\section{Ellipsoid estimation for SOC}

In section 3, we assume the state noise and the initial state are in one interval (12). As in the research field, there are other sets to be used to denote the uncertainty, such as ellipsoid. It means that interval and ellipsoid are two commonly used sets in set membership estimation. So for completeness and comparison, here in this section we consider the ellipsoid estimation for state, which corresponds to the state of charge for Lithium-ion battery.

Observing only the first state equation in equation (9) again, matrices $A, B, D$ can be identified by using least squares method. Based on this state equation, our problem is to estimate the state $x(k)$ at different time instant $k=1,2 \cdots N-1$. It is similar to section 3 that control input $u(k)$ is determined by researcher, and $u(k)$ is a deterministic value, not an uncertainty. So for convergence, we neglect this term $B u(k)$ in the latter derivation. It means that if one ellipsoid estimation for the state is derived by our own derivation, then we can apply translation transformation to give the true ellipsoid estimation for the state. Then we rewrite the considered state equation as follows.

$$
\left\{\begin{array}{l}
x(k+1)=A x(k)+D w(k) \\
x_{0}=0, k=0,1, \cdots N-1
\end{array}\right.
$$

This special state equation is driven by $w(k)$, satisfying the following norm bound.

$$
\|w(k)\| \leq 1, k=0,1, \cdots N-1
$$

Our goal here is to build ellipsoid approximation of the state recursively. Let $X_{k}$ be the set of all states where the system can be driven in time instant $k \in N$, and assume that we have build inner and outer ellipsoidal approximations $E_{i n}^{k}$ and $E_{\text {out }}^{k}$ of the $\operatorname{set} X_{k}$.

$$
E_{\text {in }}^{k} \subset X_{k} \subset E_{\text {out }}^{k}
$$

Let also

Then the set

$$
E=\left\{x=D w / w^{T} w \leq 1\right\}
$$

$$
F_{i n}^{k+1}=A E_{i n}^{k}+E=\left\{x=A w_{1}+w_{2} / w_{1} \in E_{i n}^{k}, w_{2} \in E\right\}
$$

clearly cover $X_{k+1}$, and a natural recurrent way to define an outer ellipsoidal approximation of $X_{k+1}$ is to take as $E_{\text {out }}^{k+1}$ the smallest volume ellipsoid containing $F_{\text {out }}^{k+1}$. Note that the sets $F_{i n}^{k+1}$ and $F_{\text {out }}^{k+1}$ are of the same structure: each of them is the arithmetic sum $\left\{x=w_{1}+w_{2} / w_{1} \in W_{1}, w_{2} \in W_{2}\right\}$ of two ellipsoids $W_{1}$ and $W_{2}$. Thus we come to the problem as follows: Given two ellipsoids $W_{1}$ and $W_{2}$, find the best inner and outer ellipsoidal approximations of their arithmetic sum $W_{1}+W_{2}$. In fact, it makes sense to consider a problem.

Given two ellipsoids $W_{1}$ and $W_{2}$, find the best inner and outer ellipsoidal approximations of their arithmetic sum

$$
W=\left\{x=w_{1}+w_{2} / w_{1} \in W_{1}, w_{2} \in W_{2}\right\}
$$

of two ellipsoids $W_{1}$ and $W_{2}$.

\subsection{Outer ellipsoidal approximation}

Let the ellipsoids $W_{1}$ and $W_{2}$ be represented as.

$$
W_{i}=\left\{x / x^{T} D_{i} x \leq 1\right\}
$$

Our strategy to approximate is that, we want to build a parametric family of ellipsoids in such a way that, first, every ellipsoid from the family contains the arithmetic sum $W_{1}+W_{2}$ of two given ellipsoids, and second, the problem of finding the smallest volume ellipsoid within the family is a simple problem.

Let us start with the observation that an ellipsoid.

$$
W[Z]=\left\{x / x^{T} Z x \leq 1\right\}
$$


contains $W_{1}+W_{2}$ if and only if the following implication holds.

$$
\begin{aligned}
& \underbrace{\left.\left\{x^{i}\right\}_{i=1}^{2} /\left[x^{i}\right]^{T} D_{i}\left[x^{i}\right] \leq 1, i=1,2\right\}}_{\mathbb{\Downarrow}} \\
& \left(x^{1}+x^{2}\right)^{T} Z\left(x^{1}+x^{2}\right) \leq 1
\end{aligned}
$$

Let $D^{i}$ be one block diagonal matrix, such that all diagonal blocks, except the $i$ th one, are zero, let $M[Z]$ be that.

$$
D^{1}=\left[\begin{array}{cc}
D_{1} & 0 \\
0 & 0
\end{array}\right], D^{2}=\left[\begin{array}{cc}
0 & 0 \\
0 & D_{2}
\end{array}\right], M[Z]=\left[\begin{array}{ll}
Z & Z \\
Z & Z
\end{array}\right]
$$

Due to the fact that for every symmetric positive semidefinite matrix $X$ such that $\operatorname{Tr}\left(D^{i} X\right) \leq 1, i=1,2 \cdots m$, one has $\operatorname{Tr}(M[Z] X) \leq 1$. Then we arrive at the following result.

Proposition 1: Let a positive definite matrix $Z$ be such that the optimal value in the semidefinite program.

$$
\max _{X}\left\{\operatorname{Tr}(M[Z] X) / \operatorname{Tr}\left(D^{i} X\right) \leq 1, i=1,2, X \geq 0\right\}
$$

is $\leq 1$. Then the ellipsoid

$$
W[Z]=\left\{x / x^{T} Z x \leq 1\right\}
$$

contains $W_{1}+W_{2}$ of two ellipsoids $W_{i}=\left\{x / x^{T} D_{i} x \leq 1\right\}$.

The above proposition is the first step to build a parametric family of ellipsoids, which contains the arithmetic sum $W_{1}+W_{2}$. Then the second problem of finding the smallest volume ellipsoid within the parametric family can be reduced to one semidefinite program as that.

Proposition 2: Given two centered at the origin full dimensional ellipsoids.

$$
W_{i}=\left\{x / x^{T} D_{i} x \leq 1\right\}, i=1,2
$$

Let us associate with these two ellipsoids the semidefinite program.

$$
\max _{t, Z, \lambda}\left\{\begin{array}{l}
t / t \leq \operatorname{Det}^{\frac{1}{n_{x}}}(Z), \lambda_{1} D^{1}+\lambda_{2} D^{2} \geq M[Z] \\
\lambda_{1} \geq 0, \lambda_{2} \geq 0, \lambda_{1}+\lambda_{2}=1 \\
Z \geq 0
\end{array}\right\}
$$

Every feasible solution $(t, Z, \lambda)$ to this semidefinite program with positive value of the objective produces ellipsoid $W[Z]=\left\{x / x^{T} Z x \leq 1\right\}$.

which contains $W_{1}+W_{2}$, and the smallest volume ellipsoid is given by optimal solution of the semidefinite program (55).

\subsection{Inner ellipsoidal approximation}

Let us represent the given centered at the origin ellipsoids $W_{i}$ as.

$$
W_{i}=\left\{x / x=A_{i} w / w^{T} w \leq 1\right\}, i=1,2
$$

Due to the fact that an ellipsoid $E[Z]=\left\{x=Z w / w^{T} w \leq 1\right\}$ is contained in the sum $W_{1}+W_{2}$ of the ellipsoids $W_{i}$ if and only if one has.

$$
\forall x:\left\|Z^{T} x\right\|_{2} \leq \sum_{i=1}^{2}\left\|A_{i}^{T} x\right\|_{2}
$$

A natural way to generate ellipsoids satisfying equation (56) is to note that whenever matrix $X_{i}$ satisfying the following the property about its special norms.

$$
\left|X_{i}\right|=\sqrt{\lambda_{\max }\left(X_{i}^{T} X_{i}\right)}=\max _{x}\left\{\left\|X_{i} x\right\|_{2} /\|x\|_{2}\right\} \leq 1, i=1,2
$$

Then the matrix

$$
Z=Z\left\{X_{1}, X_{2}\right\}=A_{1} X_{1}+A_{2} X_{2}
$$

satisfies equation (56).

$$
\begin{aligned}
& \left\|Z^{T} x\right\|_{2}=\left\|\left[A_{1} X_{1}+A_{2} X_{2}\right] x\right\|_{2} \leq \sum_{i=1}^{2}\left\|X_{i}^{T} A_{i}^{T} x\right\|_{2} \\
& \leq \sum_{i=1}^{2} \mid X_{i}^{T}\|\| A_{i}^{T} x\left\|_{2} \leq \sum_{i=1}^{2}\right\| A_{i}^{T} x \|_{2}
\end{aligned}
$$

Thus every collection of square matrices $X_{i}$ with spectral norms not exceeding 1 produces an ellipsoid satisfying equation (56) and thus contained in $W$.

Similarly the largest volume ellipsoid within the parametric family can also be reduced to the following semidefinite program.

\section{Proposition}

3:

Let

$W_{i}=\left\{x / x=A_{i} w / w^{T} w \leq 1\right\}, A_{i}>0, i=1,2 \quad, \quad$ consider the following semidefinite program.

$\max t$

$$
\begin{aligned}
\text { subject to } t & \leq\left(\operatorname{Det}\left(\frac{1}{2} \sum_{i=1}^{2}\left[X_{i}^{T} A_{i}^{T} x\right]\right)\right)^{\frac{1}{n_{x}}} ; \\
& \sum_{i=1}^{2}\left[X_{i}^{T} A_{i}^{T} x\right] \geq 0 ; \\
& {\left[\begin{array}{cc}
I_{n} & -X_{i}^{T} \\
-X_{i} & I_{n}
\end{array}\right] \geq 0, i=1,2 }
\end{aligned}
$$


(60)

with design variables $X_{1}, X_{2}$. Every feasible solution $\left(X_{1}, X_{2}, t\right)$ to this problem produces the ellipsoid.

$$
E\left(X_{1}, X_{2}\right)=\left\{x=\left(\sum_{i=1}^{2} A_{i} X_{i}\right) w / w^{T} w \leq 1\right\}
$$

contained in the arithmetic sum $W_{1}+W_{2}$ of the original ellipsoids, and the largest volume ellipsoid which can be obtained in this way is associated with optimal solution to the semidefinite program (60).

After solving these two semidefinite programs (55) and (60), then we build inner and outer ellipsoidal approximations $E_{\text {in }}^{k}$ and $E_{\text {out }}^{k}$ of the set $X_{k}$, i.e.

$$
E_{\text {in }}^{k} \subset X_{k} \subset E_{\text {out }}^{k}
$$

Based on these two inner and outer ellipsoidal approximations $E_{i n}^{k}$ and $E_{\text {out }}^{k}$ of the set $X_{k}$, then the final state estimation at time instant $x(k)$ can be chosen as the midpoint between the two centers of inner and outer ellipsoidal approximations $E_{i n}^{k}$ and $E_{\text {out }}^{k}$.

So generally whatever the state noise or external noise is included in an interval or an ellipsoid, firstly we apply our mentioned interval estimation or ellipsoid estimation to obtain the state estimation set,. Secondly the center or midpoint can be chosen as the final state estimation value, which corresponds to the state of charge for Lithium-ion battery.

\section{Simulation example}

Here we do not have yet the experimental platform, so this simulation example is based on references in the open literatures. To acquire experimental data such as current, voltage and temperature from the battery, a battery test bench was established. The configuration of the battery test bench is shown in Figure 6.

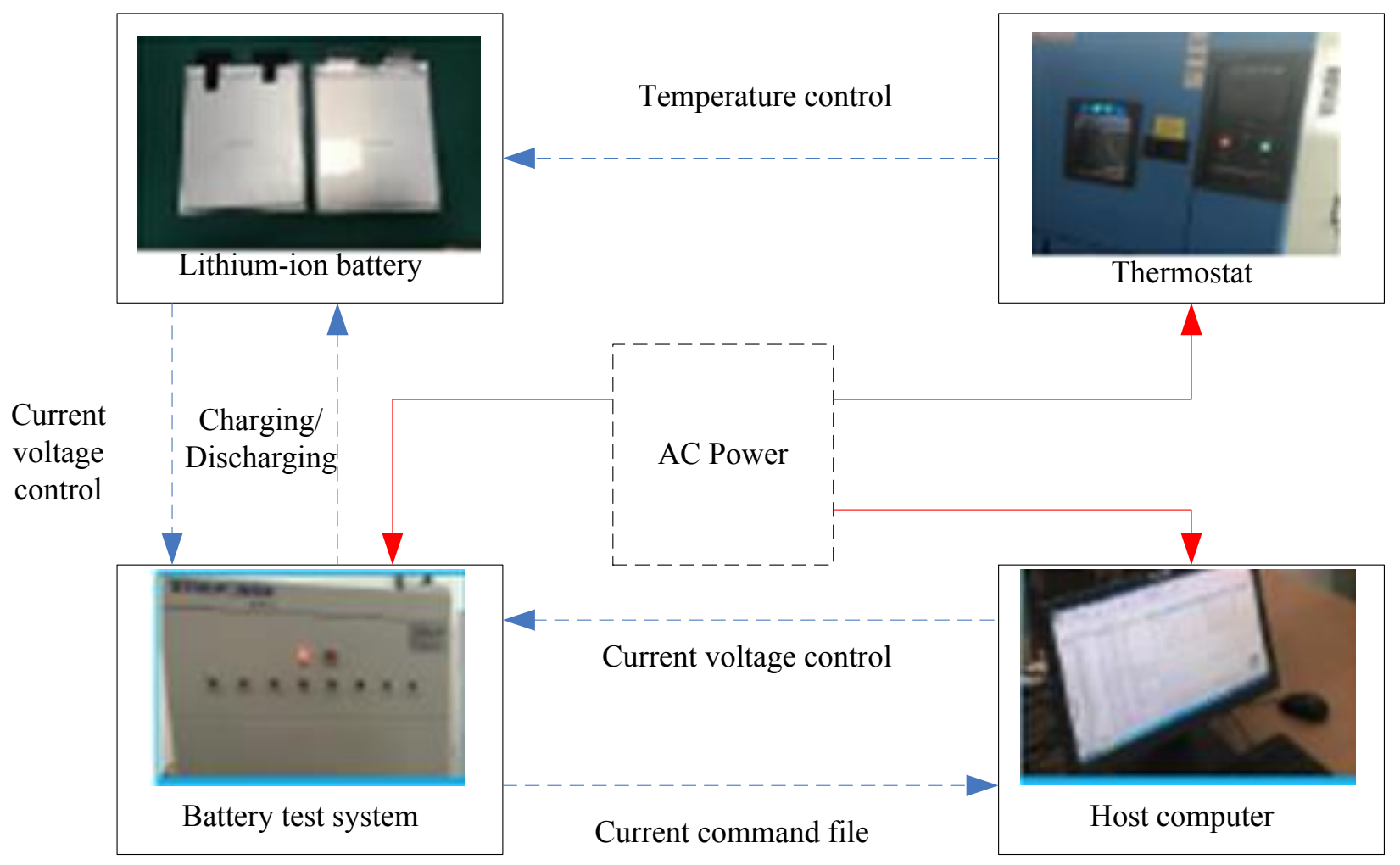

Figure 6. Lithium battery test platform 
For convenience in the latter simulation example, Lithium battery test needs to charge and discharge the lithium ion battery at different temperatures and different rates. Therefore, the equipment required for the experimental bench includes a thermostat, a battery charging and discharging device, a ternary neon battery, and a host computer. Lithium battery test platform is plotted in Figure 6, where the detailed processes are described as follows.

Step1. The charging and discharging positive and negative terminals of the battery are respectively connected to the positive and negative electrodes of the battery through the wire harness, and the wire harness of the appropriate diameter is selected according to the allowable charging and discharging ratio of the battery to avoid the burning of the wire harness. One end of the voltage sampling line to the other end of the battery is connected to the voltage sampling and wiring port of the battery charging and discharging device. Finally, the temperature measuring line of the thermistor is attached to the surface of the battery, and the other side of the temperature detecting line is connected to the temperature detecting terminal of the battery charging and discharging device.
Step 2. Set the lithium battery in the incubator, and set the experimental ambient temperature.

Step 3. Start battery charging and discharging equipment and incubator.

Step 4. In the online machine, we edit the charge and discharge test step or import the edited current test file into the host computer to automatically generate the test step, then set the sampling time and output file save address, start the test.

Based on the experimental platform, the open-circuit voltage of the battery has a monotonic relationship with the state of charge. The relation between open-circuit voltage and state of charge is established by running test on the considered lithium-ion battery. Let all batteries are fully charged and rested for 3 hours, such that the internal chemical reactions attain a desired equilibrium state. Moreover the discharge test includes a sequence of pulse current of $1 \mathrm{C}$ with 6-min discharge and 10-min rest, then the discharge test can make the battery to return back to its expected equilibrium state before running the next cycle, which is shown in Figure 7. 


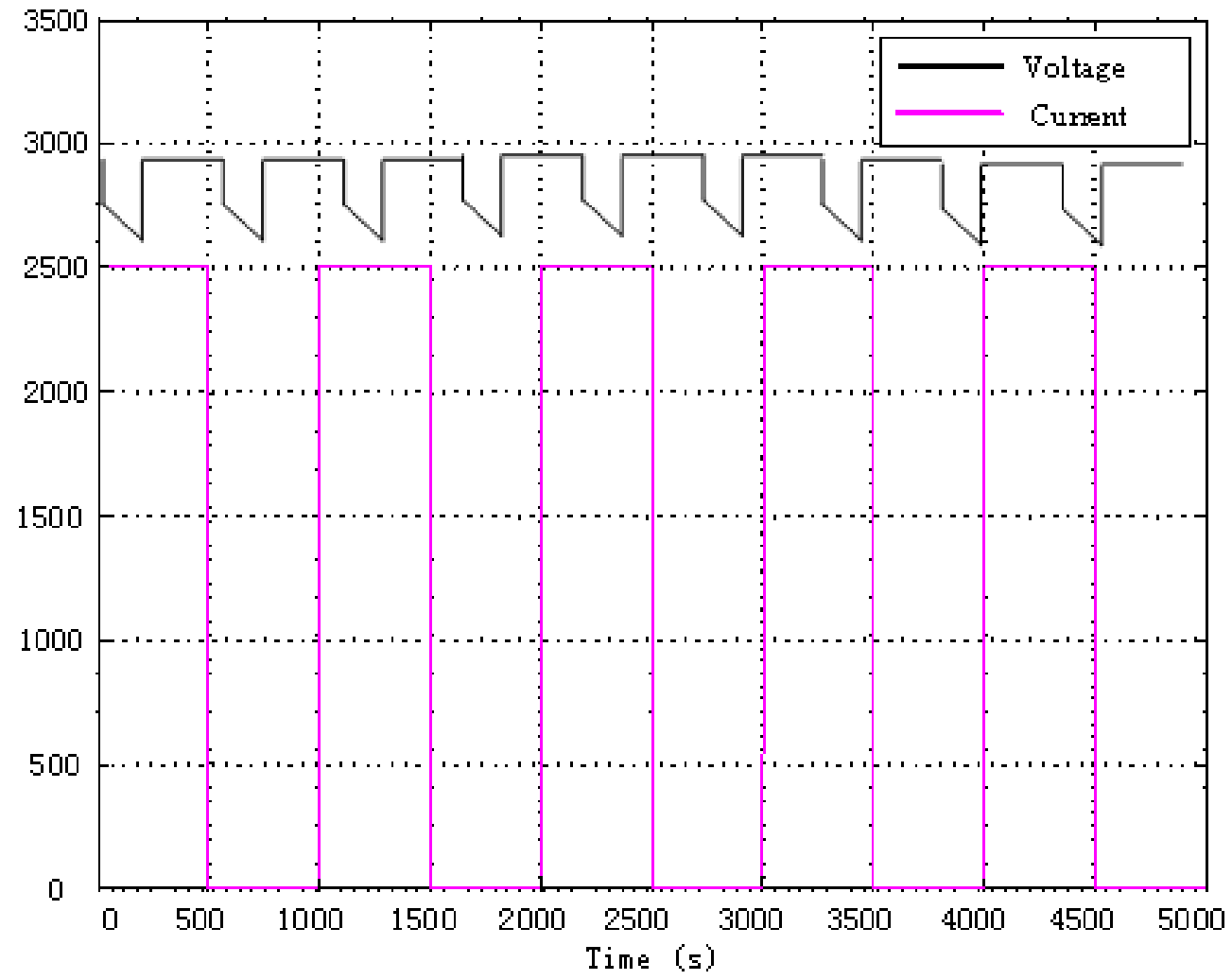

Figure 7. Voltage and current curves

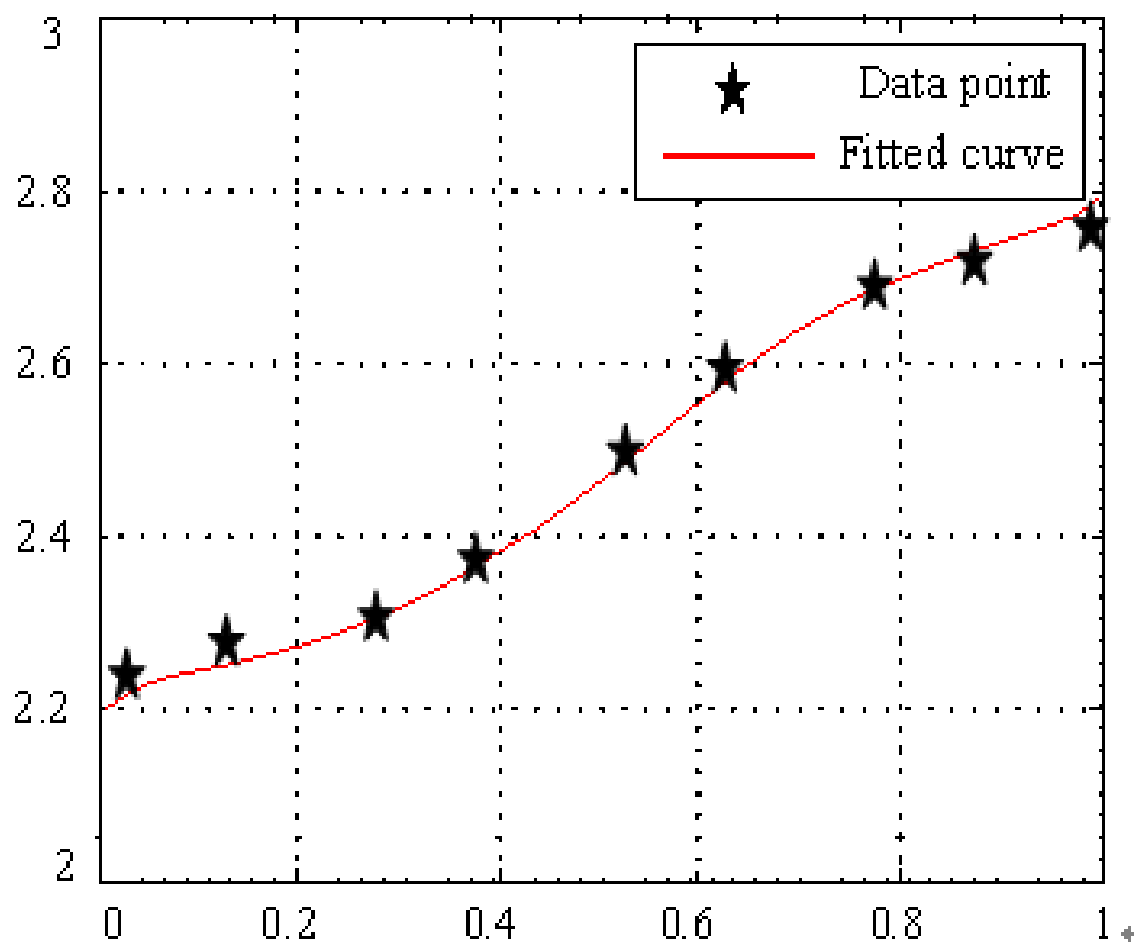

Figure 8. Polynomial form for $U_{O C}$ 
As $U_{O C}$ is rewritten as the following polynomial form $U_{O C}(x)=d_{5}+d_{4} x+d_{3} x^{2}+d_{2} x^{3}+d_{1} x^{4}$. To identify these unknown parameters in this polynomial form, Least squares method is used to achieve this goal. Then the identification result for this polynomial form is given in Figure 8, which shows the relation between the true data point and its identified polynomial form.

In the whole simulation process, the true parameters can be identified by using some system identification strategy, for example least squares method, instrumental variable method, maximum likelihood method etc. Then identified parameter are obtained as follows.

$$
\begin{aligned}
& R_{0}=0.0994 \Omega, R_{p}=0.030 \Omega, C_{p}=20773 K F ; \\
& I=1.10 A ; T_{s}=0.3 s
\end{aligned}
$$

Substituting the above values into the equation (7), (8) and (9), then each matrix is given as follows.

$$
A=\left[\begin{array}{ccc}
1 & 0 & 0 \\
0 & 0.68 & 0 \\
0 & 0 & 1
\end{array}\right], B=\left[\begin{array}{c}
-0.8 \\
0.064 \\
0.1
\end{array}\right], C=\left[\begin{array}{lll}
2.5 & -1 & 1.2
\end{array}\right], D=1
$$

Consider the unknown but bounded noise in simulation, these two kinds of unknown but bounded signals in equation (9) are formulated as.

$$
\left\{\begin{array}{l}
-0.5 \leq x(0) \leq 0.5 \\
1 \leq w(k) \leq 1 \\
1 \leq v(k) \leq 1
\end{array} \quad \text { for all } k \in R^{+}\right.
$$

In simulation we consider not only the state estimation, but also the output estimation, and the state estimation corresponds to the state of charge. The interval estimation on observed output with bounded noise $v(k)$ can be used in another research field, such as robust control, interval model predictive control etc.

Firstly we apply equation (27) to obtain the interval estimation for state. The state trajectory can be easily obtained by using equation (19) in Matlab, where some priori information about initial state and bounded noise are used. The simulation results are shown in Figure 9, where the black curve is the true state trajectory and the two red curves denote the estimated curves. One curve is consisted by upper bound, and the other curve is the lower bound. From Figure 9, we see that the true state trajectory lies in between the two red curves., so at each time instant, the midpoint of the upper bound and lower bound can be chosen as the final state estimation at the considered time instant. Similarly the interval estimation for output is also given in Figure 10, where the true output trajectory lies in between the two estimated curves. The interval estimation for output is obtained based on equation (44), and the final output estimation can also selected as the midpoint at each time instant.

For the sake of completeness, the ellipsoid estimation for state or state of charge is given in Figure 11, where the true state trajectory is same with that curve in Figure 9. Twelve data points are sampled in the true state trajectory, and we need to construct twelve ellipsoids to include these twelve data points as their own interior points. As ellipsoid is used to denote the uncertainty, so at every time instant we will obtain one ellipsoid to include the true data point. From the simulation result in Figure 11, twelve ellipsoids are constructed through using relations (47), and these twelve ellipsoids include these twelve data points as their own interior points exactly. When the center of the corresponding ellipsoid is chosen as the final state estimation, we find that the error exists yet, i.e. the center of the considered ellipsoid is not equal to the true state value at the considered time instant. 


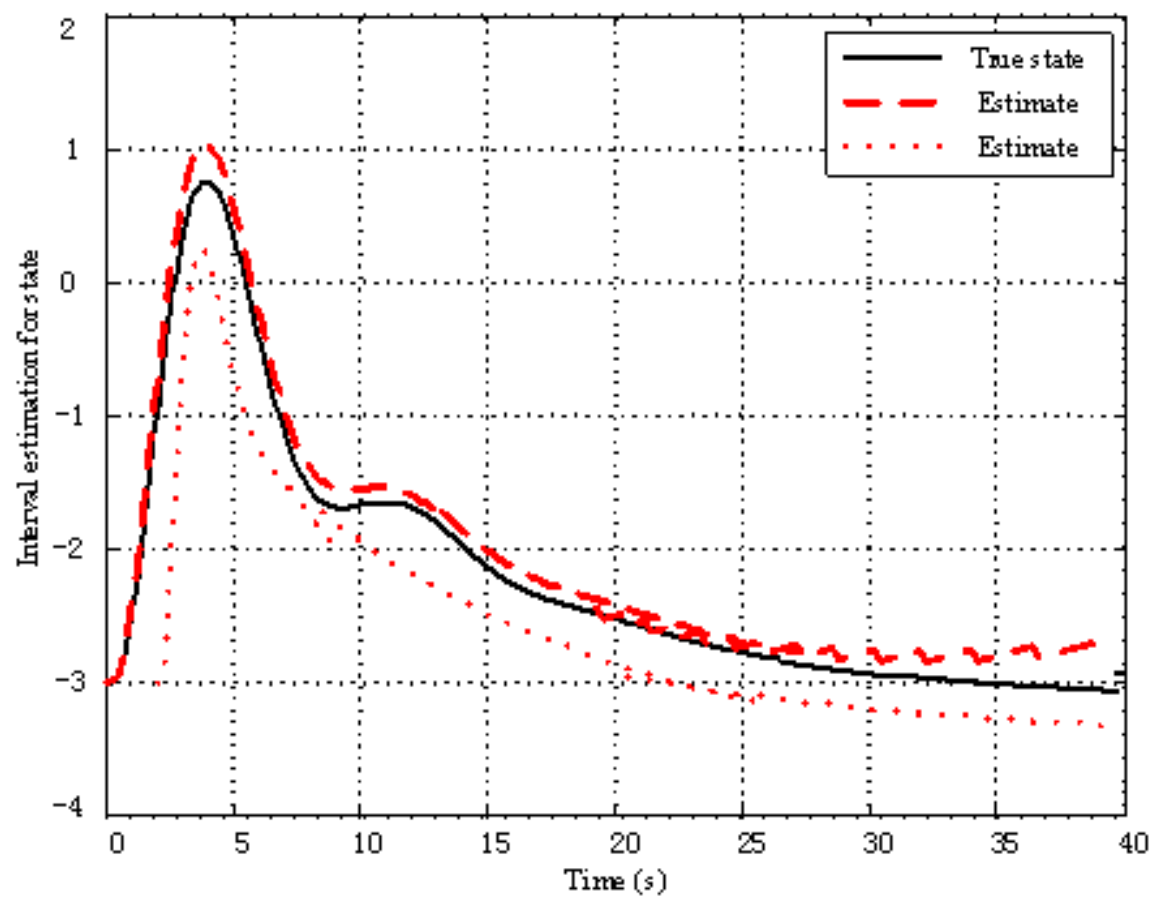

Figure 9. Interval state estimation

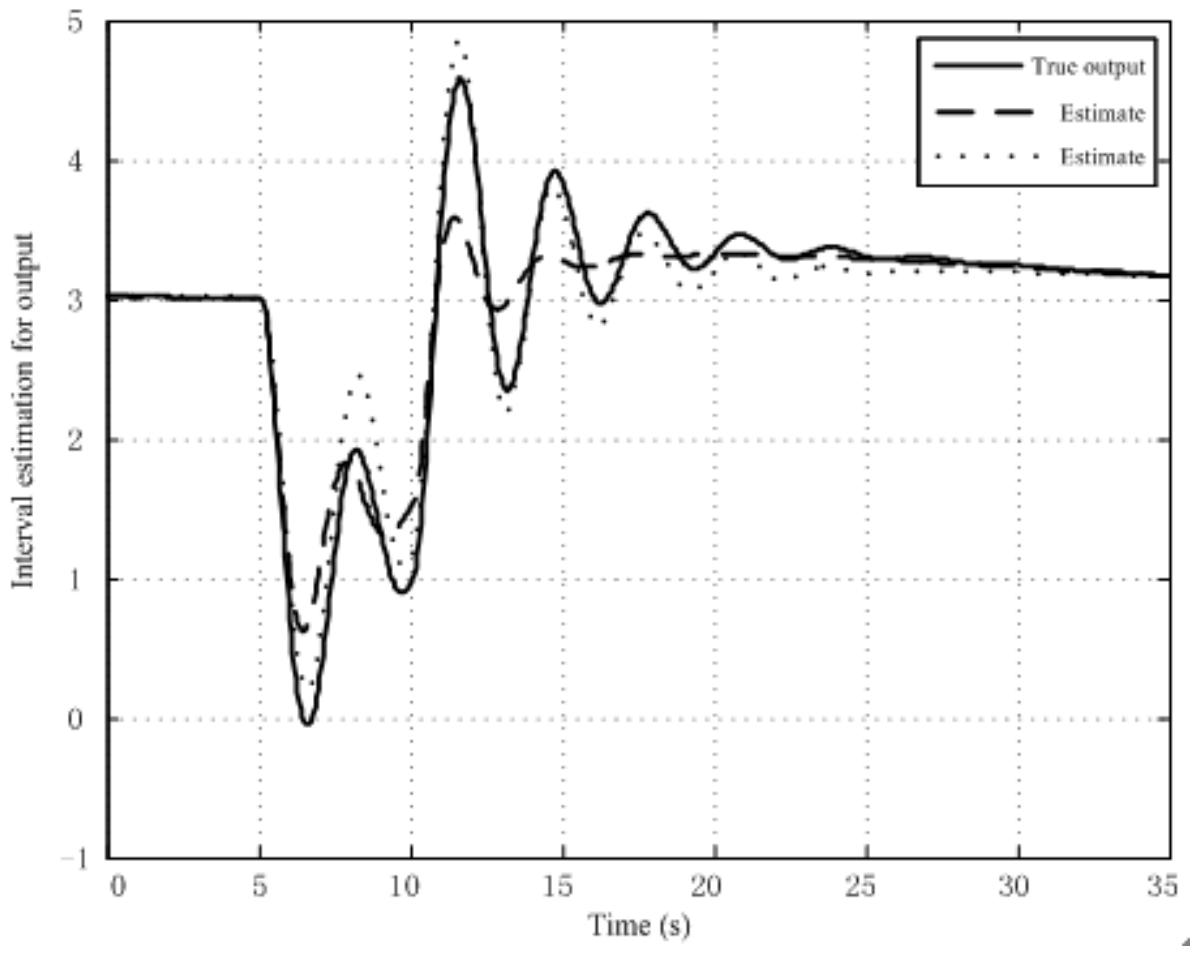

Figure 10. Interval output estimation 


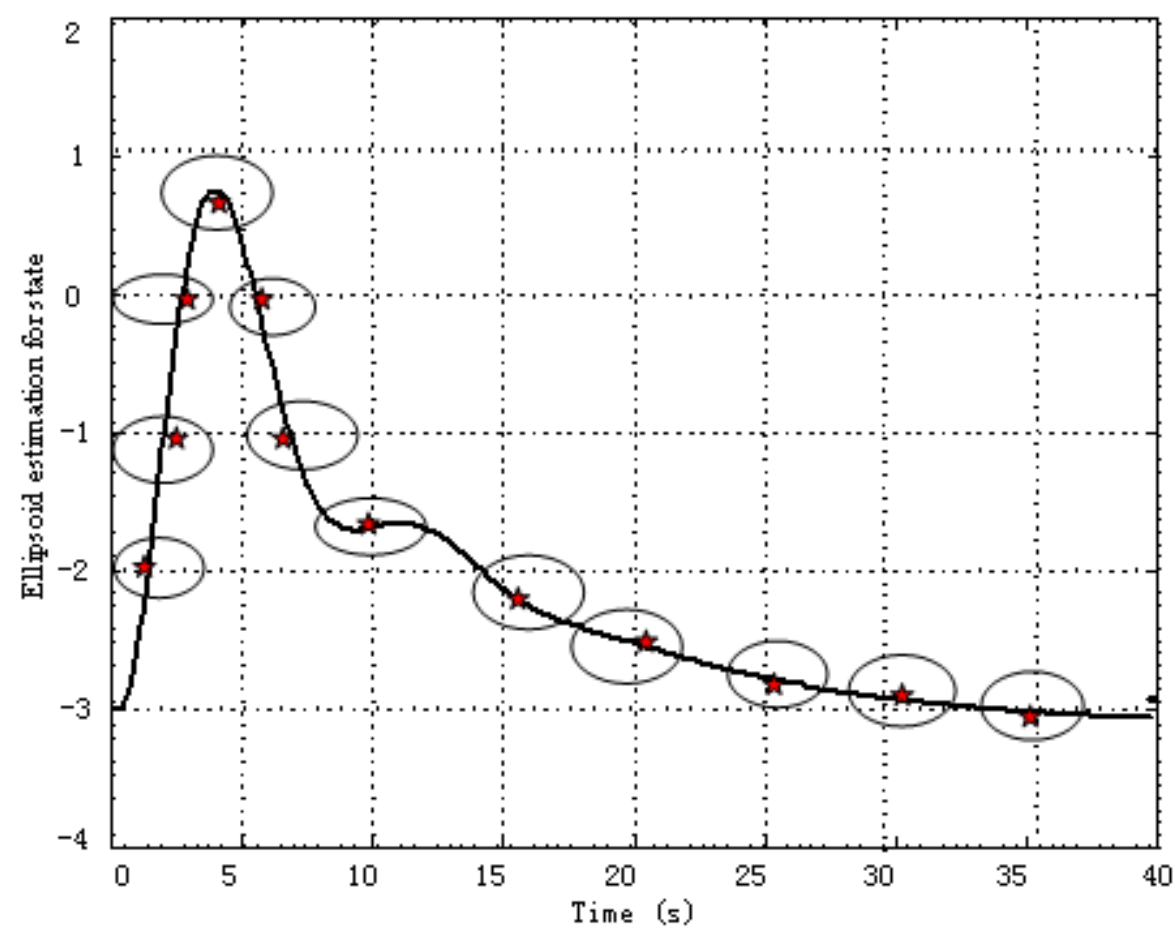

Figure 11. Ellipsoid state estimation

20202BAL202009).

\section{Conclusion}

In this paper, set membership strategy is applied to estimate the state of charge for Lithium-ion battery, so that the state estimation can be dealt with in case of unknown but bounded external noise. The goal of introducing set membership strategy is to alleviate the shortcoming of the traditional Kalman filter algorithm. After formulating one state space equation for the state of charge estimation, through using one equivalent circuit model to replace the considered state of charge estimation for Lithium-ion batter. According to the commonly used interval and ellipsoid for the external noise, the interval estimation and ellipsoid estimation are derived for the considered state estimation respectively, which corresponds to the state of charge estimation for Lithium-ion battery. But here we only propose the detailed strategy, and the accuracy or convergence of our considered strategy can be regarded as our future work.

\section{Acknowledgements}

This work is partially supported by the Grants from the Mexico National Science Foundation (No.

\section{Data Availability}

The data used to support the findings of this study are available from the corresponding author upon request.

\section{Conflict of interest}

The authors declare that there is no conflict of interests regarding the publication of this paper.

\section{References}

[1] Mohamed MR, Leung PK (2015). Performance characterization of a vanadium redox of battery at different operating parameters under a standardized test-bed system. Apply Energy 137, 402-412.

[2] Guarnieri Massimo (2016). Vanadium redox of batteries: Potentials and challenges of an emerging storage technology. IEEE Ind. Electron. Mag. 10, 20-31.

[3] Hong WC, Li BY, Wang BG (2015). Theoretical and technological aspects of ow batteries: Measurement. Energy Storage Sci. Technol. 56, 744-756.

[4] Petchsingh C, Quill N, Joyce JT, et al (2016). Spectroscopic measurement of state of charge 
in vanadium ow batteries with an analytical model of VIV-VV absorbance. J Electrochem Soc 163, 5068-5083.

[5] Li X, Xiong J, Tang A, et al(2018). Investigation of the use of electrolyte viscosity for online state-of-charge monitoring design in vanadium redox of battery. SAppl Energy 211, 1050-1059.

[6] Ngamsai Kittima, Amornchai Arpornwichanop (2015). Measuring the state of charge of the electrolyte solution in a vanadium redox flow battery using a four-pole cell device. J Power Sources 29, $150\{157$.

[7] Ressel S, Bill F, Holtz L, et al (2018). State of charge monitoring of vanadium redox of batteries using half cell potentials and electrolyte density. J Power Sources 37, 776-783.

[8] Chou YS, Hsu NY, Jeng KT, et al (2016). Et al a novel ultrasonic velocity sensing approach to monitoring state of charge of vanadium redox flow battery. Apply Energy 182, 283-289.

[9] Zhong Q, Zhong F, Cheng J, et al (2016). State of charge estimation of lithiumion batteries using fractional order sliding mode observer. ISA Trans 66, 448-459.

[10] Xiong B, Zhao J, Su Y, et al(2017). State of charge estimation of vanadium redox of battery based on sliding mode observer and dynamic model including Capacity fading factor. IEEE Trans. Sustainable Energy 8, 1658-1667.

[11] Wei Z, Tseng KJ, Wai N, et al(2017). An adaptive model for vanadium redox of battery and its application for online peak power estimation. J Power Sources 344, 195-207.

[12] Wei Z, Tseng KJ, Wai N, et al(2016). Adaptive estimation of state of charge and capacity with online identified battery model for vanadium redox of battery. J Power Sources 332, 389-398.

[13] Wei Z, Bhattaraia A, Zou C, et al (2018). Real-time monitoring of capacity loss for vanadium redox ow battery. J Power Sources 390, 261-269.

[14] Lin, C, Mu, H, Xiong, R (2017). A novel multi-model probability battery state of charge estimation approach for electric vehicles using H-infinity algorithm. Apply Energy 344, 195-207.

\section{Creative Commons Attribution License 4.0 (Attribution 4.0 International, CC BY 4.0)}

This article is published under the terms of the Creative Commons Attribution License 4.0

https://creativecommons.org/licenses/by/4.0/deed.en_US 